\title{
Illustrative Cases from the Pathfinder Clinical Trials of Patients with Hemophilia A Treated with Turoctocog Alfa Pegol (N8-GP)
}

\author{
Robert Klamroth (D' \\ Kingsley Hampton ${ }^{2}$ \\ Sonata Saulyte Trakymienè (iD) ${ }^{3}$ \\ Lars Korsholm ${ }^{4}$ \\ Manuel Carcao (iD) ${ }^{5}$ \\ 'Department of Internal Medicine, \\ Hemophilia Treatment Centre, Vivantes \\ Klinikum im Friedrichshain, Berlin, Germany; \\ ${ }^{2}$ Department of Cardiovascular Science, \\ University of Sheffield, Sheffield, UK; ${ }^{3}$ Clinic \\ of Children's Diseases, Faculty of Medicine, \\ Vilnius University, Vilnius University Hospital \\ Santaros Klinikos, Vilnius, Lithuania; \\ ${ }^{4}$ Biostatistics, Novo Nordisk A/S, Bagsværd, \\ Denmark; ${ }^{5}$ Division of Haematology/ \\ Oncology, Department of Paediatrics, The \\ Hospital for Sick Children, Toronto, Ontario, \\ Canada
}

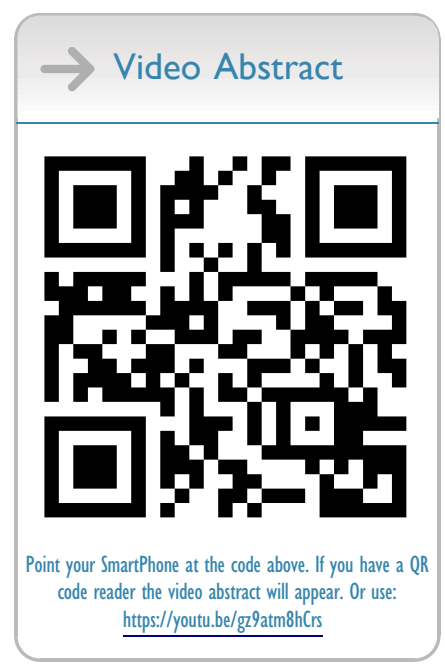

Correspondence: Robert Klamroth Department of Internal Medicine, Hemophilia Treatment Centre, Vivantes Klinikum im Friedrichshain, Berlin, Germany

Tel +4930130231575

Fax $+4930 \quad 130232130$

Email robert.klamroth@vivantes.de
Purpose: To illustrate the benefits of the extended half-life (EHL) recombinant factor VIII product N8-GP (Esperoct ${ }^{\circledR}$, turoctocog alfa pegol) by describing individual cases of patients with severe hemophilia A treated with N8-GP in the pathfinder clinical trial program.

Patients and Methods: This manuscript presents selected patient cases from the pivotal pathfinder clinical trial program, which included a number of clinical studies in adults (pathfinder 2 and 3) and children (pathfinder 5); overall results published previously. Clinical data and outcomes described in this manuscript are more detailed and derived from several interesting patient cases (five adults from pathfinder 2 and two children from pathfinder 5), who received N8-GP as prophylaxis (PPX) for their severe hemophilia A. Three of the five adults described here also underwent multiple major surgeries (for which they moved from pathfinder 2 into pathfinder 3 and later returned to pathfinder 2). New analyses on pediatric joint health from pathfinder 5 are also summarized here. Outcomes assessed included bleeding complications, improvements in quality of life, intraoperative hemostatic response, blood loss during surgery, number of blood transfusions, and annualized bleeding rates. For the pediatric patients, target joint resolution, adverse events, and annualized joint bleeding rate were also assessed, all by the treating physician.

Results: Considerable improvements in treatment adherence, bleeding rates, and overall physical activity levels were demonstrated in two adult cases from the pathfinder 2 trial. N8-GP demonstrated good or excellent hemostatic coverage in three adult patients undergoing multiple major surgeries. The benefits of N8-GP for joint health and in support of children and adolescents with evolving active lifestyles were reported for several pediatric cases.

Conclusion: These patient cases highlight the benefits of EHL products, such as N8-GP, for patients with severe hemophilia A. They include more challenging scenarios relating to improvements in previously poor adherence to PPX, children with active sporting lifestyles, and patients requiring multiple major surgeries.

Keywords: extended half-life, factor VIII, prophylaxis, surgery, joint health

\section{Plain Language Summary}

Hemophilia $\mathrm{A}$ is a rare condition that results in affected people bleeding too easily. Bleeds can damage joints and make surgery risky. Replacement clotting factors can prevent bleeds but must be injected directly into patients' veins several times per week. Over the past 10 years, newer types of clotting factor have been developed which require less frequent injections. Turoctocog alfa pegol (also known as N8-GP or Esperoct ${ }^{\mathbb{B}}$ ) is one of these treatments and has been shown in large clinical trials to be an effective treatment for hemophilia A. 
In this article, we have put together information on a selection of individual patients (five adults and seven children) treated with N8-GP. We report results for two adults who had previously struggled to take clotting factors regularly. Following a switch to N8-GP they were able to maintain their schedule of injections and experienced improvements in their levels of physical activity. We also report results for three adults who were treated with N8-GP whilst undergoing several major surgeries. For all operations, the patients were protected against excessive blood loss. Avoiding early damage to joints is important for the long-term health of individuals with hemophilia A. Here, we report results for five children with improvements in joint symptoms following regular treatment with N8-GP. Finally, we describe the experience of two children who were able to engage in sports whilst receiving N8-GP. These results highlight the potential benefits and clinical significance of utilizing N8-GP in both adults and children with hemophilia A.

\section{Introduction}

Prophylactic factor VIII (FVIII) replacement therapy has been the standard of care for patients with hemophilia A. Due to their relatively short plasma half-life (range 10-14 hours), standard half-life (SHL) recombinant FVIII (rFVIII) products require frequent intravenous injections, placing a substantial treatment burden on patients. ${ }^{1}$ To reduce the number of injections required, several rFVIII products with an extended half-life (EHL) have been developed over the past 10 years, ${ }^{1}$ including N8-GP (Esperoct $^{\circledR}$, turoctocog alfa pegol, Novo Nordisk, Bagsværd, Denmark), a glycoPEGylated rFVIII product. N8-GP has a half-life approximately 1.6-fold longer than SHL FVIII products and therefore requires less-frequent dosing. $^{2-4}$

In the pivotal Phase III pathfinder 2 and 5 trials, N8-GP demonstrated a good safety and efficacy profile when administered as prophylaxis (PPX) or as an on-demand (OD) treatment for bleeds in adults, adolescents, and children with severe hemophilia A. Patients who participated in pathfinder 2 and 5 were then eligible to enroll in the recently completed, long-term extension study, pathfinder 8 (NCT03528551).

In pathfinder 2 (NCT01480180), previously treated patients aged $\geq 12$ years were treated with N8-GP as PPX either every fourth day (Q4D, $n=177$ ) or once per week ( $n=61$ over the course of the main phase and extension) note that several patients changed treatment regimen during the trial so may be included in more than one treatment arm but counted once in the total. The median treatment duration on any regimen was 5.4 years. For patients receiving N8-GP Q4D, the median and mean annualized bleeding rates (ABRs) were 0.84 and 2.14, respectively, and the proportion of patients who experienced zero bleeds increased with every additional year of PPX - from $39.1 \%$ in the first year of treatment to $64.4 \%$ in the sixth year. Across the treatment arms, $83.2 \%$ of bleeds were successfully treated (defined as "excellent" or "good" response). In total, $80.9 \%$ of bleeds were treated with one injection and $94.9 \%$ with up to two injections. ${ }^{5}$

In pathfinder 5 (NCT01731600), previously treated patients aged $<12$ years $(n=68)$ were treated with N8-GP (50-75 IU/kg twice per week; dosing every third day was permitted based on the patient's bleeding pattern) for a median time of $\sim 4.9$ years. The median and mean ABRs were 0.81 and 1.08, respectively, and the proportion of patients who experienced zero bleeds increased with every additional year of PPX - from $32 \%$ in the first year of treatment to $70 \%$ during year 5. N8-GP treatment was successful in treating $81.6 \%$ of bleeds in all patients and most bleeds $(88.2 \%)$ were treated with $\leq 2$ injections. ${ }^{6}$ Treatment with N8-GP also resulted in improvements in joint health in patients enrolled in pathfinder 5. Overall, 12 patients had 16 target joints (TJs) at baseline, which all resolved (according to both the International Society on Thrombosis and Haemostasis [ISTH] criteria and the more stringent protocol definition) during the trial. ${ }^{6}$

N8-GP has also been shown to be effective for the perioperative management of bleeds in patients $\geq 12$ years $(n=35)$ with severe hemophilia A undergoing major surgery in the Phase III pathfinder 3 trial (NCT01489111) a sub-study of pathfinder 2. ${ }^{7,8}$ Hemostasis was successful in 47 out of $49(95.9 \%)$ major surgeries, with the remaining two surgeries defined as having a "moderate" response. A lower frequency of N8-GP injections was administered during the overall post-operative period compared with previous data for SHL rFVIII products. ${ }^{8}$

This manuscript aims to describe illustrative patient cases from the pivotal pathfinder clinical trials of previous poor adherence to PPX (cases 1 and 2), multiple ( $\geq 3$ ) major surgeries (cases 3-5), and children with active lifestyles (cases 11 and 12). In addition, we provide details of pediatric patients with TJs at enrollment (cases 6-10). Full data for these studies have been previously published, ${ }^{5,6,8}$ and here we examine some of the potential benefits of N8-GP in patients with specific challenges drawn from these largescale studies. 


\section{Patients and Methods Study Design}

All cases presented here were part of the pathfinder 2, 3, and 5 studies. The eligibility criteria and study design for pathfinder 2, 3, and 5 have been described in detail previously. ${ }^{2,3,7,9}$ All trials were approved by independent ethics committees and institutional review boards and conducted in accordance with the Declaration of Helsinki and Good Clinical Practice guidelines. No additional institutional review approval was required from the authors' institutions to publish the case details reported here. Written informed consent was obtained from all patients (and each child's legal guardian for patients under the age of 18) prior to any trial-related activities to have the case details published. The informed consent obtained from patients met the correct regulatory requirements of each investigator's respective institution or country. No patient images were used. Clinical narratives and patient data from the period prior to enrollment in the pathfinder trials were provided by the treating physician. All patient cases are from the pathfinder studies and only study information is used. All patients signed informed consent in the studies and all pathfinder studies had IRB approval. So, additional IRB approval concerning this manuscript is not necessary.

Outcomes reported for the adult cases 1 and 2 (pathfinder 2) included bleeding complications and qualitative improvements in quality of life (changes in physical activity). Whilst undergoing multiple major surgeries, cases 3-5 transferred from pathfinder 2 to pathfinder 3 . Outcomes reported for these patients included intraoperative hemostatic response, blood loss during surgery (as estimated by the surgeon), number of blood transfusions, N8-GP consumption, and adverse events (AEs). The intraoperative hemostatic response was defined as excellent (better than expected), good (as expected), moderate (less than optimal but no change in treatment regimen required), or none (bleeding due to inadequate response; regimen change required). ${ }^{7,8}$ ABRs were also calculated for the pathfinder 3 patients based on the number of bleeds reported by the end of their participation in pathfinder 2 .

Outcomes reported for the pediatric patient cases 6-10 (pathfinder 5) include TJ resolution and AEs. TJs were defined as $\geq 3$ bleeds in a consecutive 6-month period. TJ resolution was determined using either the per-protocol definition of no bleeds in a consecutive 12-month period $^{10}$ or the ISTH definition of $\leq 2$ bleeds/consecutive 12-month period. ${ }^{11}$ For pediatric patient cases 11 and 12 (pathfinder 5) we report bleeding complications, qualitative improvements in quality of life (changes in physical activity), and AEs. For pathfinder 5, other outcomes include overall joint health (proportion of patients with zero joint bleeds per year of PPX), TJ ABR, and annualized joint bleeding rate (AjBR; for each year of the study). TJ ABR were analyzed using a Poisson regression model on number of bleeding episodes per patient allowing for over-dispersion and using log planned observation duration as an offset. Estimates of the TJ ABR were provided with $95 \%$ confidence intervals.

Individual FVIII trough levels for patients (assessed by a product-specific chromogenic assay) were calculated as geometric means from all relevant visits starting from visit 3 .

\section{Results}

\section{N8-GP Treatment in Adults: Illustrative Patient Cases \\ Adults Receiving N8-GP PPX \\ Patient Case I}

This patient (Figure 1A) previously received treatment with cryoprecipitate and plasma-derived FVIII (pdFVIII), but in 2003 switched to rFVIII. Twelve months prior to pathfinder 2 enrollment, he was mainly receiving SHL rFVIII as an OD treatment, and some PPX, although his adherence to PPX was poor with only one treatment administered each week. During this time, the patient experienced multiple bleeds; with all of this he developed arthropathy of the right knee and both ankles. According to the treating physician, the patient's motivation for entering pathfinder 2 was to receive PPX with a reduced number of injections.

During pathfinder 2, this patient was noted to have excellent adherence to PPX and a marked reduction in the number of bleeding episodes were reported. Overall, the patient experienced six bleeds during pathfinder 2 compared with 35 bleeds during the 12 months prior to enrollment, resulting in substantial reductions in ABR (35 to 1 ). The FVIII trough level of this patient was $2.2 \%$. The patient also reported a significant improvement in physical activity, albeit with some continued impairment due to hemophilic arthropathy.

\section{Patient Case 2}

The second patient from pathfinder 2 (Figure 1B) had previously received treatment with cryoprecipitate and then OD pdFVIII. In 2004, he moved to twice weekly rFVIII PPX, where his adherence was noted to be poor. 
A

\begin{tabular}{|c|c|c|c|c|}
\hline $\begin{array}{l}\text { Patient Profile } \\
\text { (Baseline) }\end{array}$ & Age: 40 years old & BMI: 25.1 & Body weight: $76.0 \mathrm{~kg}$ & Race: White \\
\hline
\end{tabular}

Before switch

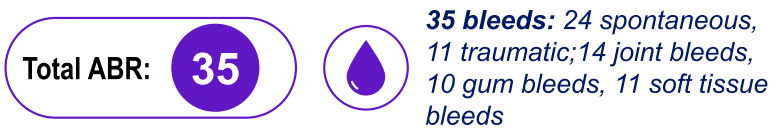
Little sport (cycling, fishing)

\section{After switch}

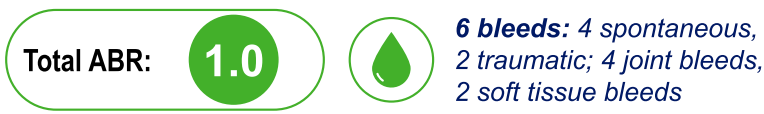

Significant increase in sporting activity

OD SHL rFVIII

B

\begin{tabular}{|c|c|c|c|c|}
\hline $\begin{array}{l}\text { Patient Profile } \\
\text { (Baseline) }\end{array}$ & Age: 41 years old & BMI: 24.7 & Body weight: $74.0 \mathrm{~kg}$ & Race: White \\
\hline
\end{tabular}

\section{Before switch}

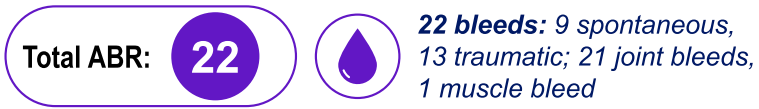
1 muscle bleed

\section{After switch}

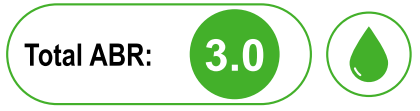

13 bleeds: 8 spontaneous, 5 traumatic; 10 joint bleeds, 3 soft tissue bleeds

\section{No sporting activity}

Significant increase in sporting activity

\begin{tabular}{lll}
2012 On pathfinder 2: N8 -GP PPX \\
\hline SHL rFVIII PPX \\
to PPX \\
\hline Excellent adherence to PPX
\end{tabular}

Figure I Illustrative patient cases from pathfinder 2. (A) Patient case I; (B) Patient case 2.

Notes: For 12 months prior to enrollment in pathfinder 2, patient I received $26 \mathrm{IU} / \mathrm{kg} \mathrm{SHL} \mathrm{rFVIII} \mathrm{as} \mathrm{OD} \mathrm{treatment} \mathrm{and} \mathrm{patient} 2$ received I3 IU/kg PPX Q4D. During the pathfinder program, both patients received $50 \mathrm{IU} / \mathrm{kg}$ N8-GP Q4D (pathfinder 2) or three times weekly (pathfinder 8). During pathfinder 2, a trough levels of 2.2\% was reported for patient I and $1.9 \%$ for patient 2 .

Abbreviations: ABR, annualized bleeding rate; BMI, body mass index; BW, body weight; OD, on demand; PPX, prophylaxis; Q4D, every 4 days; rFVIII, recombinant factor VIII; SHL, standard half-life.

In the 12 months prior to enrollment in pathfinder 2, his treatment regimen was $\mathrm{rFVIII}$ PPX Q4D, on which he experienced multiple bleeds, including 21 separate joint bleeds - mostly in the right elbow and right hip. His relevant past medical history included hepatitis B and $\mathrm{C}$ infections and replacement of the left knee and right 
hip. Like case 1, the patient's motivation for entering pathfinder 2 was to receive PPX with a reduced number of injections.

During pathfinder 2, the patient's ABR reduced considerably to 3 compared with 22 prior to enrollment; his FVIII trough level was $1.9 \%$. As per patient 1 , this patient was able to significantly increase his sporting activity during pathfinder 2. As expected, the high dose of N8-GP (50 IU/kg Q4D) during pathfinder 2 was more efficient than the $13 \mathrm{IU} / \mathrm{kg}$ Q4D dose of SHL product the patient received prior to pathfinder 2 .

\section{Adults Undergoing Multiple Major Surgeries}

Patient Cases 3-5

Details of the three adults who underwent $\geq 3$ major surgeries are shown in Figure 2A-C. These patients transferred from pathfinder 2 to pathfinder 3 for the period during which they had multiple major surgeries. Following completion of each surgery, the patients resumed prophylactic N8-GP as part of pathfinder 2. Patient case 3 underwent three elective procedures. For the first surgery, a laparotomy and pseudotumor excision, blood loss was slightly higher than anticipated (2 L vs $1.5 \mathrm{~L}$ ) and two blood transfusions were required. For the other two surgeries, blood loss was no greater than anticipated $(0.2 \mathrm{~L}$ vs $0.3 \mathrm{~L}$ for right total knee replacement [TKR] and $0.18 \mathrm{~L}$ for both anticipated and estimated blood loss for left TKR) and no transfusions were required. Patient case 4 underwent two elective and two emergency procedures. Blood loss of $1 \mathrm{~L}$ occurred during the emergency removal of an infected right knee prosthesis, but the volume was as anticipated. The patient required one blood transfusion for the bilateral knee prosthesis and two blood transfusions for removal of the infected right knee prosthesis. Finally, patient case 5 underwent four elective procedures. A slightly higher than anticipated volume of blood was lost during a cholecystectomy $(0.1 \mathrm{~L}$ vs $0 \mathrm{~L})$, but no transfusion was required. For all surgeries, the hemostatic response was described as good or excellent and this was maintained post surgery, with uncomplicated recoveries across all procedures. N8-GP consumption for all surgeries is presented in Table 1. All patients received a single pre-surgery dose and for four surgeries a single post-surgery dose was required on the day of surgery. For days 1-6 and 7-14 post surgery, total consumption ranged from 150.0 to $245.3 \mathrm{IU} / \mathrm{kg}$ and 53.9 to $273.6 \mathrm{IU} / \mathrm{kg}$, respectively. All three patients had an ABR of 0 at the end of pathfinder 2. In addition, during participation in pathfinder 2, FVIII trough levels were 3.8\% for patient $3,8.8 \%$ for patient 4 , and $1.1 \%$ for patient case 5 .
N8-GP was well tolerated in all five cases from pathfinder 2/3. Patient 1 experienced a moderate AE of rotator cuff syndrome; all other reported AEs were mild. All AEs were considered unrelated to treatment and no N8-GP dose adjustments were required.

\section{N8-GP Experience in Children Joint Health}

For all patients enrolled in pathfinder 5 , the overall median and mean TJ ABRs were 0.00 and 0.09 , respectively (Table 2). Overall, $20(31.7 \%)$ pediatric patients in pathfinder 5 had zero joint bleeds during the trial. As demonstrated in Figure 3, there was a trend for improved joint health for older children (aged 6-11 years) over the duration of the trial, with the proportion of older children with zero joint bleeds increasing with each successive year. Similarly, the mean AjBR decreased with each successive year of N8-GP PPX for older children, from 1.26 in year 1 to 0.42 in year 5 . For the nine older children remaining in the study in year 6 , no joint bleeds were reported (Table 2). A similar trend was reported in younger children (aged 0-5 years), although the picture was less clear, likely due to increased physical activity as these children develop.

\section{Patient Cases 6-10}

In this article, we present five patients with seven TJs at baseline who had no TJ bleeds during the duration of the trial (mean 4.5 years) (Table 3 ). Two patients were $<5$ years old and had not previously received PPX treatment. The other three were between 5 and 10 years old and had developed TJs prior to enrollment in pathfinder 5 despite PPX with SHL rFVIII products.

\section{Long-Term Pediatric Experience with N8-GP PPX: Illustrative Patient Cases}

Patient Case II

At enrollment, the patient (Figure 4A) was 11 years old. After completing the trial, he entered pathfinder 8 and is currently studying at university. Prior to pathfinder 5 enrollment, the patient was receiving SHL rFVIII twice weekly but continued to experience bleeds on this PPX regimen. Of note, his estimated FVIII trough level was $2.5 \%$ at 48 hours and, as such, it was estimated that his levels would be $<1 \%$ at 72 hours. According to the treating physician, during the 12 months prior to pathfinder 5 inclusion his $\mathrm{ABR}$ was 3, with all bleeds involving traumatic injuries to joints. Without N8-GP treatment his physician felt that he would have needed to escalate to 


\section{A}

$\begin{array}{llll}\text { Q Patient Profile (Baseline) } & \text { Age: } 50 \text { years old } & \text { BMl: } 25.0 \quad \text { Body weight: } 64.0 \mathrm{~kg} & \text { Race: Asian }\end{array}$

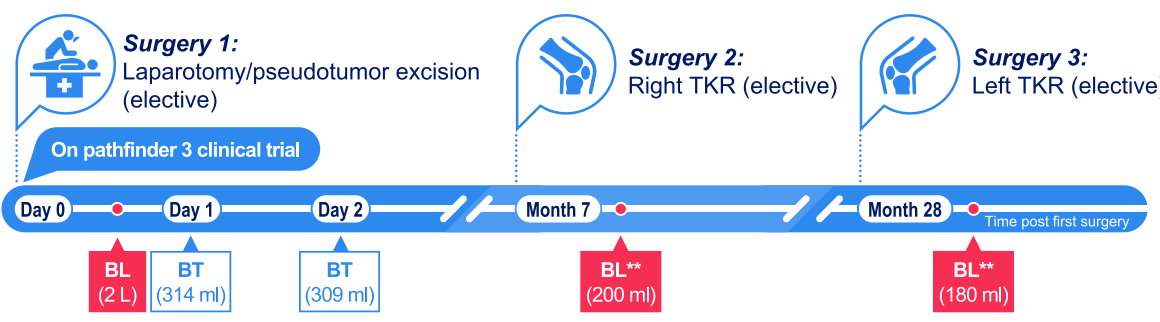

\begin{tabular}{|l|l|l|}
\hline $\begin{array}{l}\text { AE: Hematuria } \\
\text { (Day -1 pre surgery and } \\
\text { day 15 post surgery) }\end{array}$ & $\begin{array}{c}\text { AE: } \\
\text { Tooth extraction } \\
\text { Day -10 pre surgery } \\
\text { AE: } \begin{array}{l}\text { Post-procedural fever } \\
\text { Days 1-6 post surgery } \\
\text { AE: }\end{array}\end{array}$ & $\begin{array}{l}\text { Hypoesthesia } \\
\text { Days 1-6 post surgery }\end{array}$ \\
\begin{tabular}{|l|l|l|} 
AE: Blister \\
Day of surgery \\
Days 1-6 post surgery
\end{tabular} \\
\hline $\begin{array}{l}\text { Hemostatic } \\
\text { response: }\end{array}$ & Good & \\
\hline
\end{tabular}

B

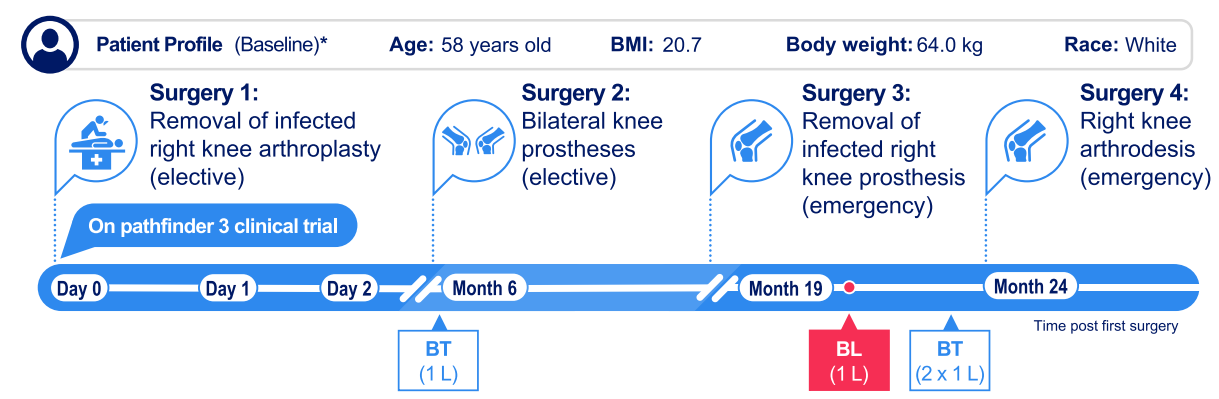

\begin{tabular}{|l|c|c|c|}
\hline $\begin{array}{l}\text { AE: Increased C-reactive } \\
\text { protein } \\
\text { (Days 1-6 post surgery) }\end{array}$ & $\begin{array}{c}\text { AE: Increased platelet count } \\
\text { Days 7-14 post surgery }\end{array}$ & $\begin{array}{c}\text { AE: } \begin{array}{l}\text { Nausea } \\
\text { Days 1-6 } \\
\text { post surgery } \\
\text { AE: }\end{array} \\
\begin{array}{l}\text { Decreased } \\
\text { hemoglobin } \\
\text { Days 1-6 post surgery }\end{array}\end{array}$ & AE: None reported \\
\hline $\begin{array}{l}\text { Hemostatic } \\
\text { Fesponse: }\end{array}$ & Excellent & Excellent 15 post surgery & Excellent \\
\hline
\end{tabular}

C

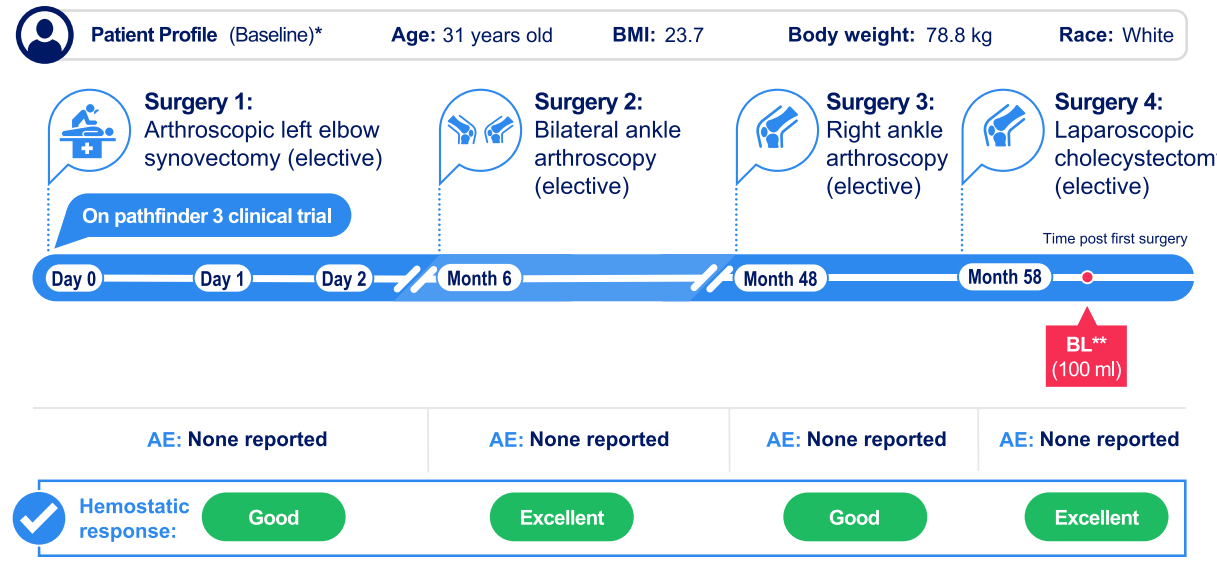

Figure 2 Illustrative patient cases from pathfinder 3. (A) Patient case 3; (B) Patient case 4; (C) Patient case 5.

Notes: *Age and weight were as reported at the pathfinder 3 screening visit. $* *$ No transfusion required.

Abbreviations: AE, adverse event; BL, blood loss; BMI, body mass index; BT, blood transfusion; TKR, total knee replacement. 
Table I N8-GP Consumption in Patients Undergoing Multiple $(\geq 3)$ Major Surgeries During Pathfinder 3

\begin{tabular}{|c|c|c|c|c|c|}
\hline & \multicolumn{4}{|c|}{ N8-GP Consumption (IU/kg) } & \multirow{2}{*}{$\begin{array}{c}\text { Pre Surgery } \\
\text { (Pre Dose) } \\
\text { FVIII Activity Value } \\
(\mathrm{U} / \mathrm{mL})^{\mathbf{b}}\end{array}$} \\
\hline & $\begin{array}{c}\text { Pre } \\
\text { Surgery } \\
\text { (Day 0) }\end{array}$ & $\begin{array}{c}\text { Post } \\
\text { Surgery } \\
(\text { Day 0) }\end{array}$ & $\begin{array}{l}\text { Post Surgery, } \\
\text { Days I-6 (Total) }\end{array}$ & $\begin{array}{c}\text { Post Surgery, } \\
\text { Days 7-14 } \\
\text { (Total) }\end{array}$ & \\
\hline \multicolumn{6}{|l|}{ Patient case 3} \\
\hline Laparotomy/pseudotumor excision & 75.0 & 50.0 & 225.0 & 192.2 & 0.892 \\
\hline Right TKR & 50.3 & 50.3 & 245.3 & 273.6 & 0.391 \\
\hline Left TKR & 50.0 & 0 & 239.4 & I59.1 & 0.653 \\
\hline \multicolumn{6}{|l|}{ Patient case 4} \\
\hline $\begin{array}{l}\text { Removal of infected right knee } \\
\text { arthroplasty }\end{array}$ & 78.1 & 0 & 195.3 & 164.1 & 0.065 \\
\hline Bilateral knee prostheses & 86.2 & 50.0 & 200.0 & 189.2 & 0.071 \\
\hline Removal of infected right knee prosthesis & 76.9 & 50.0 & 150.0 & 200.0 & 0.054 \\
\hline Right knee arthrodesis & 76.9 & 0 & 161.5 & 153.8 & 0.207 \\
\hline \multicolumn{6}{|l|}{ Patient case 5} \\
\hline Arthroscopic left elbow synovectomy & 51.2 & 0 & 209.4 & 127.2 & 0.617 \\
\hline Bilateral ankle arthroscopy & 52.6 & 0 & 163.0 & 53.9 & 0.0045 \\
\hline Right ankle arthroscopy & 51.0 & 0 & 159.2 & 106.3 & 0.0045 \\
\hline Laparoscopic cholecystectomy & 50.6 & 0 & 164.8 & 60.8 & 0.0045 \\
\hline
\end{tabular}

Notes: ${ }^{a}$ Time from end of surgery until midnight on day of surgery; ${ }^{\mathrm{b}}$ Measured by a chromogenic product-specific assay.

Abbreviation: TKR, total knee replacement.

Table 2 Target Joint (TJ) Annualized Bleeding Rate (ABR) in All Patients Receiving N8-GP Prophylaxis and Mean Annualized Joint Bleeding Rates (AjBR) by Year Over the Duration of the Pathfinder 5 Trial

\begin{tabular}{|c|c|c|c|c|c|c|}
\hline & \multicolumn{6}{|c|}{ TJ ABR } \\
\hline & \multicolumn{2}{|c|}{$\begin{array}{c}\text { Younger Children (0-5 Years) } \\
{[n=34]}\end{array}$} & \multicolumn{2}{|c|}{$\begin{array}{l}\text { Older Children (6-I I Years) } \\
\qquad[n=34]\end{array}$} & \multicolumn{2}{|r|}{$\begin{array}{c}\text { Total } \\
{[n=68]}\end{array}$} \\
\hline Mean (SD) & \multicolumn{2}{|c|}{$0.03(0.14)$} & \multicolumn{2}{|r|}{$0.15(0.38)$} & \multicolumn{2}{|r|}{$0.09(0.29)$} \\
\hline Median (IQR) & \multicolumn{2}{|c|}{$0.00(0.00-0.00)$} & \multicolumn{2}{|c|}{$0.00(0.00-0.00)$} & \multicolumn{2}{|r|}{$0.00(0.00-0.00)$} \\
\hline Range & \multicolumn{2}{|r|}{$0.00-0.78$} & \multicolumn{2}{|r|}{$0.00-1.50$} & \multicolumn{2}{|r|}{$0.00-1.50$} \\
\hline Poisson estimate mean $(95 \% \mathrm{Cl})$ & \multicolumn{2}{|c|}{$0.04(0.01-0.20)$} & \multicolumn{2}{|c|}{$0.14(0.07-0.31)$} & \multicolumn{2}{|r|}{$0.09(0.04-0.20)$} \\
\hline Trial Year & $\mathbf{n}$ & Mean AjBR (SD) & $\mathbf{n}$ & Mean AjBR (SD) & $\mathbf{n}$ & Mean AjBR (SD) \\
\hline Ist year & 29 & $0.38(0.68)$ & 34 & $1.26(1.88)$ & 63 & $0.86(\mathrm{I} .5 \mathrm{I})$ \\
\hline 2nd year & 29 & $0.21(0.49)$ & 34 & $0.79(1.57)$ & 63 & $0.52(1.23)$ \\
\hline $3 r d$ year & 29 & $0.52(0.91)$ & 34 & 0.71 (I.49) & 63 & $0.62(1.25)$ \\
\hline 4 th year & 29 & $0.07(0.26)$ & 34 & $0.50(0.99)$ & 63 & $0.30(0.78)$ \\
\hline 5 th year & 29 & $0.31(1.07)$ & 34 & $0.42(0.89)$ & 63 & $0.37(0.97)$ \\
\hline 6th year & 9 & $0.70(2.1 \mathrm{I})$ & 9 & $0.00^{\mathrm{a}}$ & 18 & 0.35 (I.49) \\
\hline
\end{tabular}

Notes: The Poisson estimate is based on a Poisson regression model with age group as a factor allowing for over-dispersion and using the log of treatment duration as an

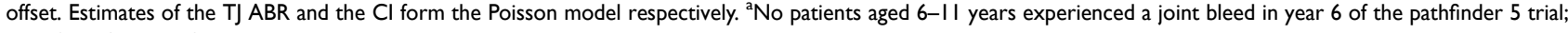
therefore, SD and IQR were not calculated.

Abbreviations: $\mathrm{Cl}$, confidence interval; IQR, interquartile range; SD, standard deviation. 


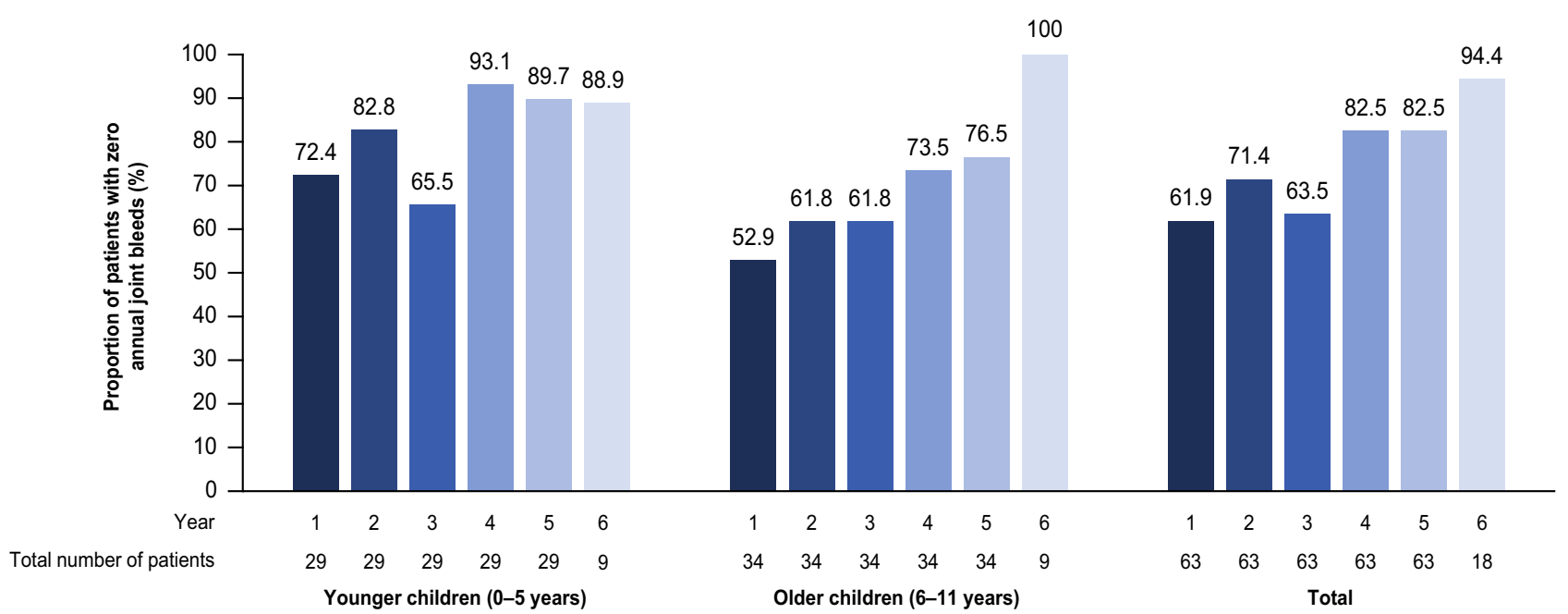

Figure 3 Patients with zero annual joint bleeds during the pathfinder 5 trial.

SHL rFVIII injections three times per week, which the patient was keen to avoid.

During the 5 years that he received N8-GP in pathfinder 5, the patient had an ABR of 1 and a trough FVIII level of $2.9 \%$. He was initially able to maintain twiceweekly injections and this supported his participation in sports. Later sports became his priority and he started to play American football and rugby. To support this lifestyle, he was escalated to three injections per week to provide higher FVIII trough levels when he transitioned to pathfinder 8 at around 16 years of age. Now at 18 years of age, he is once again less active (mainly due to COVID19 pandemic) and has reverted back to a twice-weekly regimen.

\section{Patient Case 12}

This patient (Figure 4B) was 7 years old at enrollment. Prior to enrollment in pathfinder 5 he was receiving PPX with SHL FVIII twice per week. His bleeding history included one joint bleed and during the 12 months prior to pathfinder 5 inclusion his ABR was 7 . His motivation for entering pathfinder 5 was to avoid escalating from two to three injections per week as his level of sports activities increased. Whilst participating in pathfinder 5 his ABR decreased to 1 and he had 6 bleeding episodes in total in the 5 years that he received N8-GP. His FVIII trough level was $2.8 \%$. After completing pathfinder 5 , the patient subsequently enrolled in pathfinder 8 .

This patient was able to maintain a twice-weekly dosing regimen during his entire participation in the pathfinder studies. According to his treating physician, switching from SHL rFVIII to N8-GP supported his increase in sporting activity. His family also considered the timing of the N8-GP injections (Sundays and Wednesdays) to be convenient.

For all pediatric cases described here, the safety profile of N8-GP throughout the pathfinder 5 trial was uneventful. Overall, two patients experienced a medical event of special interest. These were two episodes of allergic edema of the face (patient case 8), which was considered by the investigator to be unlikely to be related to treatment, and one accidental overdose (patient case 9 was prescribed

Table 3 Patients with Target Joints (TJs) at Baseline Who Reported No TJ Bleeds During Pathfinder 5

\begin{tabular}{|l|c|l|l|c|c|}
\hline Patient & $\begin{array}{c}\text { Age at Baseline } \\
\text { (Years) }\end{array}$ & \multicolumn{1}{|c|}{$\begin{array}{c}\text { TJ Location(s) at } \\
\text { Baseline }\end{array}$} & Prior Treatment & $\begin{array}{c}\text { If on Prior PPX, Age at Initiation } \\
\text { (Years) }\end{array}$ & $\begin{array}{c}\text { EDs Prior to } \\
\text { Trial }\end{array}$ \\
\hline Case 6 & 3 & Right knee & On-demand rFVIII & - & 5 I \\
Case 7 & 4 & Left ankle and left knee & On-demand rFVIII & - & 54 \\
Case 8 & 5 & Right knee and left knee & rFVIII PPX & 5 & 66 \\
Case 9 & 10 & Left knee & rFVIII PPX & I & 1399 \\
Case 10 & 7 & Right ankle & rFVIII PPX & I & 192 \\
\hline
\end{tabular}

Abbreviations: ED, exposure days; PPX, prophylaxis; rFVIII, recombinant factor VIII. 
A

Patient Profile Age: 11 years old $\quad$ BMI: $21.8 \quad$ Body weight: $49.3 \mathrm{~kg} \quad$ Race: White
(Baseline)

Before switch

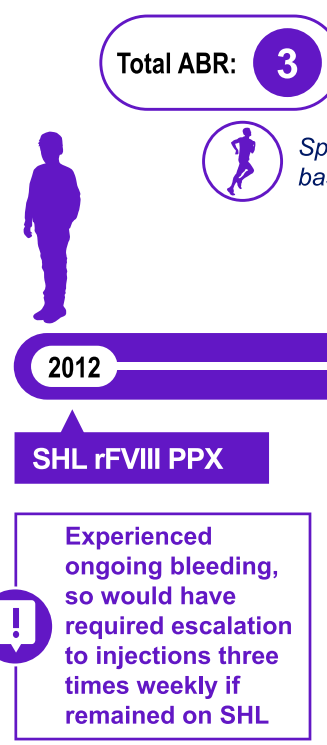

After switch

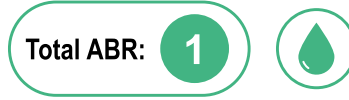

6 bleeds: 5 traumatic, 1 spontaneous;

2 joint bleeds, 2 soft tissue bleeds,

1 gum bleed, 1 other

B

Able to maintain injection frequency of twice weekly during pathfinder 5
Opted for more injections to achieve higher trough levels during pathfinder 8

Able to frequently play a large number of sports whilst at high school
Before switch

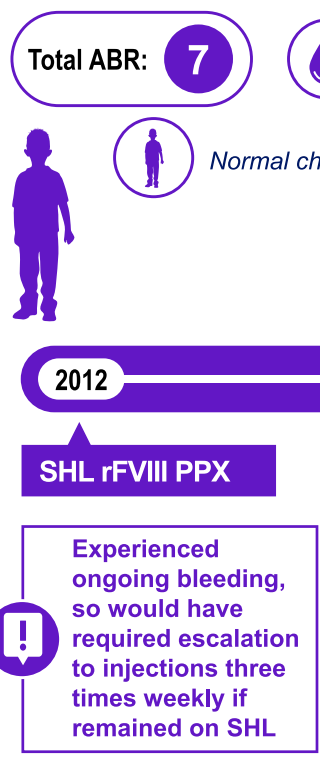

After switch

3 bleeds: all traumatic; 1 joint bleed, 2 soft tissue bleeds ood activity

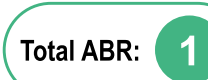

$(4)$

Played sports: track and field, and volleyball

6 bleeds: 2 traumatic, 4 spontaneous 3 joint bleeds, 3 soft tissue bleeds

Figure 4 Illustrative patient cases from pathfinder 5. (A) Patient case II; (B) Patient case I2.

Notes: Patient case II: For 12 months prior to enrollment in pathfinder 5, the patient received $40 \mathrm{IU} / \mathrm{kg}$ BW SHL rFVIII twice weekly. During the pathfinder program, the patient received N8-GP at 50-75 IU/kg BW twice weekly (pathfinder 5, FVIII trough level 2.9\%) and $50 \mathrm{IU} / \mathrm{kg}$ BW three times weekly (pathfinder 8). Patient case I2: For I2 months prior to enrollment in pathfinder 5 , the patient received $40 \mathrm{IU} / \mathrm{kg}$ BW SHL rFVIII twice weekly (factor level $2.5 \%$ at 48 hours). During the pathfinder program, the patient received N8-GP at 50-75 IU/kg BW twice weekly (pathfinder 5, FVIII trough level 2.8\%) and $50 \mathrm{IU} / \mathrm{kg}$ BW twice weekly (pathfinder 8).

Abbreviations: ABR, annualized bleeding rate; BMI, body mass index; BW, body weight; FVIII, factor VIII; PPX, prophylaxis; rFVIII, recombinant FVIII; SHL, standard half-life. 
$4 \mathrm{~mL}$ of $2000 \mathrm{IU}$ N8-GP but was dosed in error with $8 \mathrm{~mL}$ of 4000 IU N8-GP on exposure day 2). Neither event resulted in any long-term sequelae. All other reported AEs for the cases included here were mild or moderate and unrelated to treatment. All patients recovered from AEs without N8-GP dose adjustment.

\section{Discussion}

The pivotal pathfinder trials have demonstrated the longterm safety and efficacy of N8-GP in over 270 adult and pediatric patients with severe hemophilia A, including for PPX, for OD treatment of bleeds, and to support surgical procedures. $^{5,6,8}$ The pathfinder trials are the largest and longest clinical trial program conducted in hemophilia A to date, with more than 882 patient years of treatment recorded as of 2020. ${ }^{9}$ Other EHL rFVIII products such as rFVIIIFc (Elocta ${ }^{\circledR} /$ Eloctate $^{\circledR}$, Sobi, Stockholm, Sweden/ Biogen Inc, Cambridge, MA, USA), BAX 885 (Adnovi ${ }^{\circledR} /$ Adynovate $^{\circledR}$, Baxalta, a Takeda company, Lexington, MA, USA), and BAY 94-9027 (Jivi ${ }^{\circledR}$, Bayer, Whippany, NJ, USA) have also been approved for use in severe hemophilia A.

Using a simple fixed interval and fixed weight-based N8-GP dosing regimen, patients of all ages were shown to achieve higher FVIII trough levels with fewer injections when compared with SHL products. ${ }^{12,13}$ N8-GP offers patients good protection from bleeds using a twice-weekly or Q4D dosing regimen. ${ }^{5,6}$ These dosing regimens are convenient compared with more frequent regimens, as the number of infusions required on school or workdays may be reduced. ${ }^{13}$ This has practical advantages for patients and their families and may be helpful in improving adherence. The psychological benefits of reducing the number of injections, particularly for children and adolescents, should also not be underestimated and may result in improved compliance. The benefits of the potential for N8-GP to improve adherence are illustrated in the two adult cases derived from pathfinder 2. These patients had previously displayed poor adherence to PPX and both were affected by hemophilic arthropathy and limitations to their sporting activity. During pathfinder 2 , both patients displayed a marked reduction in bleeding complications and a considerable increase in their sporting activities. Both patients were reported to have excellent adherence to N8-GP, which may be due to the reduced dosing frequency and the benefits of treatment that they experienced.

N8-GP PPX can in some cases be modified to align with patients' individual needs and activity levels. ${ }^{12}$ For example, treatment with EHL products may enable patients with severe hemophilia A to have greater levels of sports participation and to take part in contact sports, which they are usually advised to avoid. ${ }^{13}$ These potential benefits are illustrated in the two long-term pediatric cases examined here. Both patients initiated N8-GP as children but received ongoing treatment supporting their transition from childhood through to adolescence and adulthood. Sports played a major role in the life of these children and, in both cases, they were able to increase their sporting activities whilst maintaining a twice-weekly dosing regimen and experiencing few bleeding episodes. Ultimately, patient case 11 increased to three doses per week to successfully support his involvement in contact sports.

In the pediatric setting, patients may commence PPX with SHL FVIII on alternate days straight away in order to optimize protection against bleeding. ${ }^{14}$ However, to avoid the use of a central venous access device, children are often initiated at a reduced frequency PPX, eg once per week, and then escalated - in most cases PPX is ultimately required every other day. ${ }^{13,14}$ For these children, switching from an SHL to an EHL rFVIII may prevent the need for escalation to every-other-day PPX, as twice-per-week EHL rFVIII PPX is likely sufficient for most children.

Careful management with rFVIII is required to manage bleeding during surgery for patients with severe hemophilia A. ${ }^{13}$ EHL products offer the advantage of convenient surgical dosing (once per day after the first 24-48 hours post surgery) and the ability to perform assays at a convenient time, eg when laboratories are available to process samples. Use of EHL products may also result in reduced factor consumption and consequently a lower cost burden. ${ }^{15}$ In all studies conducted with EHL products, hemostasis was rated as excellent or good for major and minor surgeries..$^{8,16-18}$ To date, evidence to support the use of EHL products in patients requiring multiple major surgeries has been limited to a single patient treated with BAY 94-9027. ${ }^{18}$ Here, we report three patient cases that demonstrated consistent efficacy for patients undergoing multiple major surgeries who received N8-GP, with no change in hemostatic efficacy or consumption of N8-GP reported across three or four surgeries.

Minimizing joint damage is a critical part of managing hemophilia in childhood and use of EHL products may potentially lead to higher trough levels and maintenance of long-term joint health. ${ }^{19}$ The benefits of N8-GP for the resolution of TJs have been reported previously ${ }^{6}$ and we 
examined data for five children with TJs at baseline who experienced no TJ bleeds throughout pathfinder 5. Overall, nearly one-third of patients experienced zero joint bleeds during the study, with the benefits increasing with each successive year of treatment for patients aged 6-11 years. ${ }^{6}$ These results provide evidence that early PPX with N8-GP may confer significant benefits for joint health, even for patients with pre-existing TJs.

The cases presented here illustrate some of the challenges associated with the treatment of severe hemophilia A and the potential ability of EHL products, such as N8-GP, to address these issues. ${ }^{15,20,21}$ The strength of our case series is that all on-trial data were collected systematically as part of the pathfinder program. However, as for all case series, our study is limited by selection bias and potentially recall bias for subjective outcomes.

\section{Conclusions}

These patient cases derived from the pathfinder trial program illustrate the potential benefits of N8-GP in a range of patients: adults with previously poor adherence and those undergoing multiple surgeries, and children undertaking extreme sporting activities. N8-GP has also shown positive effect on the overall joint health in children.

\section{Abbreviations}

$\mathrm{ABR}$, annualized bleeding rate; $\mathrm{AE}$, adverse event; $\mathrm{AjBR}$, annualized joint bleeding rate; $\mathrm{BL}$, blood loss; $\mathrm{BMI}$, body mass index; BT, blood transfusion; BW, body weight; CI, confidence interval; EHL, extended half-life; FVIII, factor VIII; IQR, interquartile range; ISTH, International Society on Thrombosis and Haemostasis; OD, on demand; pdFVIII, plasma-derived FVIII; PPX, prophylaxis; Q4D, every 4 days; rFVIII, recombinant FVIII; SD, standard deviation; SHL, standard half-life; TJ, target joint; TKR, total knee replacement.

\section{Acknowledgments}

Medical writing support was provided by Lindsay Uglow, $\mathrm{PhD}$, of Arc, a division of Spirit Medical Communications Group Limited and Stephanie Carter, PhD, CMPP, a contract writer working on behalf of Arc, and funded by Novo Nordisk, in accordance with Good Publication Practice 3 (GPP3) guidelines (www.ismpp.org/gpp3). The authors would like to thank Dr Ekaterina Gresko (Novo Nordisk) for her contribution to the development of the manuscript.

\section{Disclosure}

Robert Klamroth reports having received speaker bureau honoraria from Novo Nordisk, Bayer, BioMarin, Biotest, CSL Behring, Octapharma, Pfizer, Roche, Sobi, and Shire/Takeda, and honoraria for consultancy from Novo Nordisk, Bayer, BioMarin, Octapharma, Pfizer, Roche, and Shire/Takeda. Kingsley Hampton reports having received lecturing and advisory board honoraria from Novo Nordisk. Sonata Saulyte Trakymienè received speaker bureau honoraria from Novo Nordisk, Bayer, Roche, and Shire/Takeda. Lars Korsholm is an employee of Novo Nordisk. Manuel Carcao reports having received research support from Novo Nordisk, Bayer, Bioverativ/Sanofi, CSL-Behring, Octapharma, Pfizer, and Shire/Takeda. He has also received honoraria for speaking/ participating in advisory boards from Novo Nordisk, Bayer, Bioverativ/Sanofi, Biotest, CSL Behring, Grifols, LFB, Octapharma, Pfizer, Roche, and Shire/Takeda. The authors report no other conflicts of interest in this work.

\section{References}

1. Mannucci PM. Hemophilia therapy: the future has begun. Haematologica. 2020;105(3):545-553. doi:10.3324/haematol.2019.232132

2. Giangrande P, Andreeva T, Chowdary P, et al. Clinical evaluation of glycoPEGylated recombinant FVIII: efficacy and safety in severe haemophilia A. Thromb Haemost. 2017;117(2):252-261. doi:10.1160/TH16-060444

3. Meunier S, Alamelu J, Ehrenforth S, et al. Safety and efficacy of a glycoPEGylated rFVIII (turoctocog alpha pegol, N8-GP) in paediatric patients with severe haemophilia A. Thromb Haemost. 2017;117 (9):1705-1713. doi:10.1160/TH17-03-0166

4. Tiede A, Brand B, Fischer R, et al. Enhancing the pharmacokinetic properties of recombinant factor VIII: first-in-human trial of glyco-PEGylated recombinant factor VIII in patients with hemophilia A. J Thromb Haemost. 2013;11(4):670-678. doi:10.1111/ jth. 12161

5. Giangrande P, Abdul Karim F, Nemes L, et al. Long-term safety and efficacy of N8-GP in previously treated adults and adolescents with hemophilia A: final results from pathfinder 2. J Thromb Haemost. 2020;18(Suppl 1):5-14. doi:10.1111/jth.14959.

6. Šaulytė Trakymienė S, Economou M, Kenet G, Landorph A, Shen C, Kearney S. Long-term safety and efficacy of N8-GP in previously treated pediatric patients with hemophilia A: final results from pathfinder 5. J Thromb Haemost. 2020;18(Suppl 1):15-25. doi:10.1111/ jth. 15036

7. Hampton K, Chowdary P, Dunkley S, et al. First report on the safety and efficacy of an extended half-life glycoPEGylated recombinant FVIII for major surgery in severe haemophilia A. Haemophilia. 2017;23(5):689-696. doi:10.1111/hae.13246

8. Tosetto A, Neff A, Lentz SR, et al. Turoctocog alfa pegol provides effective management for major and minor surgical procedures in patients across all age groups with severe haemophilia A: full data set from the pathfinder 3 and 5 phase III trials. Haemophilia. 2020;26 (3):450-458. doi:10.1111/hae.13980

9. Matsushita T, Mangles S. An overview of the pathfinder clinical trials program: long-term efficacy and safety of N8-GP in patients with hemophilia A. J Thromb Haemost. 2020;18(Suppl 1):26-33. doi: $10.1111 /$ jth. 14958 
10. Meunier S, D'oiron R, Chambost H, Dolimier E, Guillet B. ORTHem 15-25 Study Group. Choice of factor VIII/IX regimen in adolescents and young adults with severe or moderately severe haemophilia. A French national observational study (ORTHem 15-25). Thromb Res. 2017;151:17-22. doi:10.1016/j.thromres.2016.12.023

11. Blanchette VS, Key NS, Ljung LR, et al. Definitions in hemophilia: communication from the SSC of the ISTH. J Thromb Haemost. 2014;12(11):1935-1939. doi:10.1111/jth.12672

12. Chowdary P, Carcao M, Holme PA, et al. Fixed doses of N8-GP prophylaxis maintain moderate-to-mild factor VIII levels in the majority of patients with severe hemophilia A. Res Pract Thromb Haemost. 2019;3(3):542-554. doi:10.1002/rth2.12220

13. Srivastava A, Santagostino E, Dougall A, et al. WFH guidelines for the management of hemophilia, 3rd edition. Haemophilia. 2020;26 (Suppl 6):1-158. doi:10.1111/hae.14046

14. Rayment R, Chalmers E, Forsyth K, et al. Guidelines on the use of prophylactic factor replacement for children and adults with haemophilia A and B. Br J Haematol. 2020;190(5):684-695. doi:10.1111/ bjh. 16704

15. Chowdary P. Extended half-life recombinant products in haemophilia clinical practice - expectations, opportunities and challenges. Thromb Res. 2020;196:609-617. doi:10.1016/j.thromres.2019.12.012

16. Mahlangu JN, Ragni M, Gupta N, et al. Long-acting recombinant

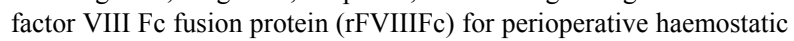
management in severe haemophilia A. Thromb Haemost. 2016;116 (1):1-8. doi:10.1160/TH15-10-0780
17. Gruppo R, López-Fernández M-F, Wynn TT, Engl W, Sharkhawy M, Tangada S. Perioperative haemostasis with full-length, PEGylated, recombinant factor VIII with extended half-life (rurioctocog alfa pegol) in patients with haemophilia A: final results of a multicentre, single-arm phase III trial. Haemophilia. 2019;25(5):773-781. doi:10.1111/hae.13807

18. Santagostino E, Lalezari S, Reding MT, et al. Safety and efficacy of BAY 94-9027, an extended-half-life factor VIII, during surgery in patients with severe hemophilia A: results of the PROTECT VIII clinical trial. Thromb Res. 2019;183:13-19. doi:10.1016/j. thromres.2019.08.023

19. Lambert T, Benson G, Dolan G, et al. Practical aspects of extended half-life products for the treatment of haemophilia. Ther $A d v$ Hematol. 2018;9(9):295-308. doi:10.1177/2040620718796429

20. Mannucci PM, Cortesi PA, Di Minno MND, Sanò M, Mantovani LG, Di Minno G. Comparative analysis of the pivotal studies of extended half-life recombinant FVIII products for treatment of haemophilia A. Haemophilia. 2021;27(4):e422-e433. doi:10.1111/hae.14313

21. Brennan Y, Parikh S, McRae S, Tran H. The Australian experience with switching to extended half-life factor VIII and IX concentrates: on behalf of the Australian Haemophilia Centre Directors' Organisation. Haemophilia. 2020;26(3):529-535. doi:10.1111/ hae. 13970
Patient Preference and Adherence

\section{Publish your work in this journal}

Patient Preference and Adherence is an international, peer-reviewed, open access journal that focusing on the growing importance of patient preference and adherence throughout the therapeutic continuum. Patient satisfaction, acceptability, quality of life, compliance, persistence and their role in developing new therapeutic modalities and compounds to optimize clinical outcomes for existing disease

\section{Dovepress}

states are major areas of interest for the journal. This journal has been accepted for indexing on PubMed Central. The manuscript management system is completely online and includes a very quick and fair peer-review system, which is all easy to use. Visit http:// www.dovepress.com/testimonials.php to read real quotes from published authors. 